\title{
Enzymatic Machinery for Endocannabinoid Biosynthesis Associated with Calcium Stores in Glutamatergic Axon Terminals
}

\author{
Rita Nyilas, ${ }^{1}$ Barna Dudok, ${ }^{1}$ Gabriella M. Urbán, ${ }^{1}$ Ken Mackie, ${ }^{2}$ Masahiko Watanabe, ${ }^{3}$ Benjamin F. Cravatt ${ }^{4}$ \\ Tamás F. Freund, ${ }^{1}$ and István Katona ${ }^{1}$ \\ ${ }^{1}$ Institute of Experimental Medicine, Hungarian Academy of Sciences, H-1083 Budapest, Hungary, ${ }^{2}$ Department of Psychological and Brain Sciences, \\ Indiana University, Bloomington, Indiana 47405, ${ }^{3}$ Department of Anatomy, Hokkaido University School of Medicine, Sapporo 060-8638, Japan, and ${ }^{4}$ The \\ Skaggs Institute for Chemical Biology and Department of Chemical Physiology, The Scripps Research Institute, La Jolla, California 92037
}

\begin{abstract}
Endocannabinoids are regarded as retrograde signaling molecules at various types of synapses throughout the CNS. The lipid derivatives anandamide and 2-arachidonoylglycerol (2-AG) are generally thought to be the key molecular players in this process. Previous anatomical and electrophysiological studies provided compelling evidence that the biosynthetic enzyme of 2-AG is indeed localized in the postsynaptic plasma membrane, whereas its target, the $\mathrm{CB}_{1}$ cannabinoid receptor, and the enzyme responsible for its inactivation are both found presynaptically. This molecular architecture of 2-AG signaling is a conserved feature of most synapses and supports the retrograde signaling role of 2-AG. Conversely, the molecular and neuroanatomical organization of synaptic anandamide signaling remains largely unknown. In contrast to its predicted role in retrograde signaling, here we show that $\mathrm{N}$-acylphosphatidylethanolaminehydrolyzing phospholipase D (NAPE-PLD), a biosynthetic enzyme of anandamide and its related bioactive congeners, the $\mathrm{N}$-acylethanolamines (NAEs), is concentrated presynaptically in several types of hippocampal excitatory axon terminals. Furthermore, high-resolution quantitative immunogold labeling demonstrates that this calcium-sensitive enzyme is localized predominantly on the intracellular membrane cisternae of axonal calcium stores. Finally, the highest density of NAPE-PLD is found in mossy terminals of granule cells, which do not express $\mathrm{CB}_{1}$ receptors. Together, these findings suggest that anandamide and related NAEs are also present at glutamatergic synapses, but the sites of their synthesis and action are remarkably different from 2-AG, indicating distinct physiological roles for given endocannabinoids in the regulation of synaptic neurotransmission and plasticity.
\end{abstract}

Key words: cannabinoid; NAPE-PLD; anandamide; glutamatergic synapse; calcium store; mossy terminal

\section{Introduction}

Recent advances in lipidomics highlighted the presence of an unexpected repertoire of lipid-derived signaling molecules in the brain (Piomelli et al., 2007). One of the best-known examples of these endogenous neuroactive lipids are the so-called endocannabinoids. Although the molecular species belonging to this group are structurally heterogeneous, most mimic some of the characteristic physiological effects evoked by biologically active compounds of the cannabis plant (Di Marzo et al., 2007). In an attractive paradigm for their precise modus operandi in the ner-

Received Nov. 16, 2007; revised Dec. 17, 2007; accepted Dec. 19, 2007.

This work was supported by Hungarian Scientific Research Fund (OTKA) Grants T046820 (T.F.F.) and F046407 (I.K.), Hungarian Health Science Council (ETT) Grant 561/2006 (I.K.), European Union Contract LSHM-CT-2004005166 (T.F.F.), Hungarian National Office for Research and Technology (NKFP) Grant 1A/002/2004 (T.F.F.), National Institutes of Health Grants DA11322 and DA21696 (K.M.), and by the Howard Hughes Medical Institute. István Katona is a grantee of the János Bolyai scholarship. We are grateful to Drs. Andrea Lörincz, Viktor Varga, László Acsády, and Csaba Fekete for their insightful advice and to Dr. Balázs Hangya and Anikó Ludányi for help with the statistical analysis. We also thank Katalin Lengyel, Katalin Iványi, Emőke Simon, and Győző Goda for excellent technical assistance and Drs. Jin Fu and Daniele Piomelli for the NAPE-PLD construct.

Correspondence should be addressed to Dr. István Katona, Institute of Experimental Medicine, Hungarian Academy of Sciences, H-1083 Budapest, Szigony utca 43, Hungary. E-mail: katona@koki.hu.

DOI:10.1523/JNEUROSCI.5102-07.2008

Copyright $\odot 2008$ Society for Neuroscience $\quad$ 0270-6474/08/281058-06\$15.00/0 vous system, endocannabinoids are released from postsynaptic neurons in an activity-dependent manner and travel retrogradely through the synaptic cleft and engage presynaptic $\mathrm{CB}_{1}$ cannabinoid receptors, which then suppress neurotransmitter release from axon terminals (Wilson and Nicoll, 2002). Importantly, variations in this basic scheme account for numerous forms of short- and long-term synaptic plasticity and experiencedependent modifications of neuronal activity in the CNS from the spinal cord to the neocortex (Chevaleyre et al., 2006). The major endocannabinoid responsible for retrograde synaptic communication is proposed to be 2-arachidonoylglycerol (2AG) (Kim and Alger, 2004; Makara et al., 2005; Hashimotodani et al., 2007). In contrast to the physiological role of 2-AG, the cellular mechanisms of endogenous signaling pathways mediated by other endocannabinoids remain obscure.

Among these endogenous cannabimimetic signaling molecules, anandamide ( $N$-arachidonoylethanolamine) stands out as the first identified and most intensively studied (Devane et al., 1992). Anandamide is closely related to a sizeable family of bioactive lipids, the $\mathrm{N}$-acylethanolamines (NAEs), with whom it shares common metabolic pathways for generation and inactivation in neurons (Di Marzo et al., 1994). A broad physiological and 
A

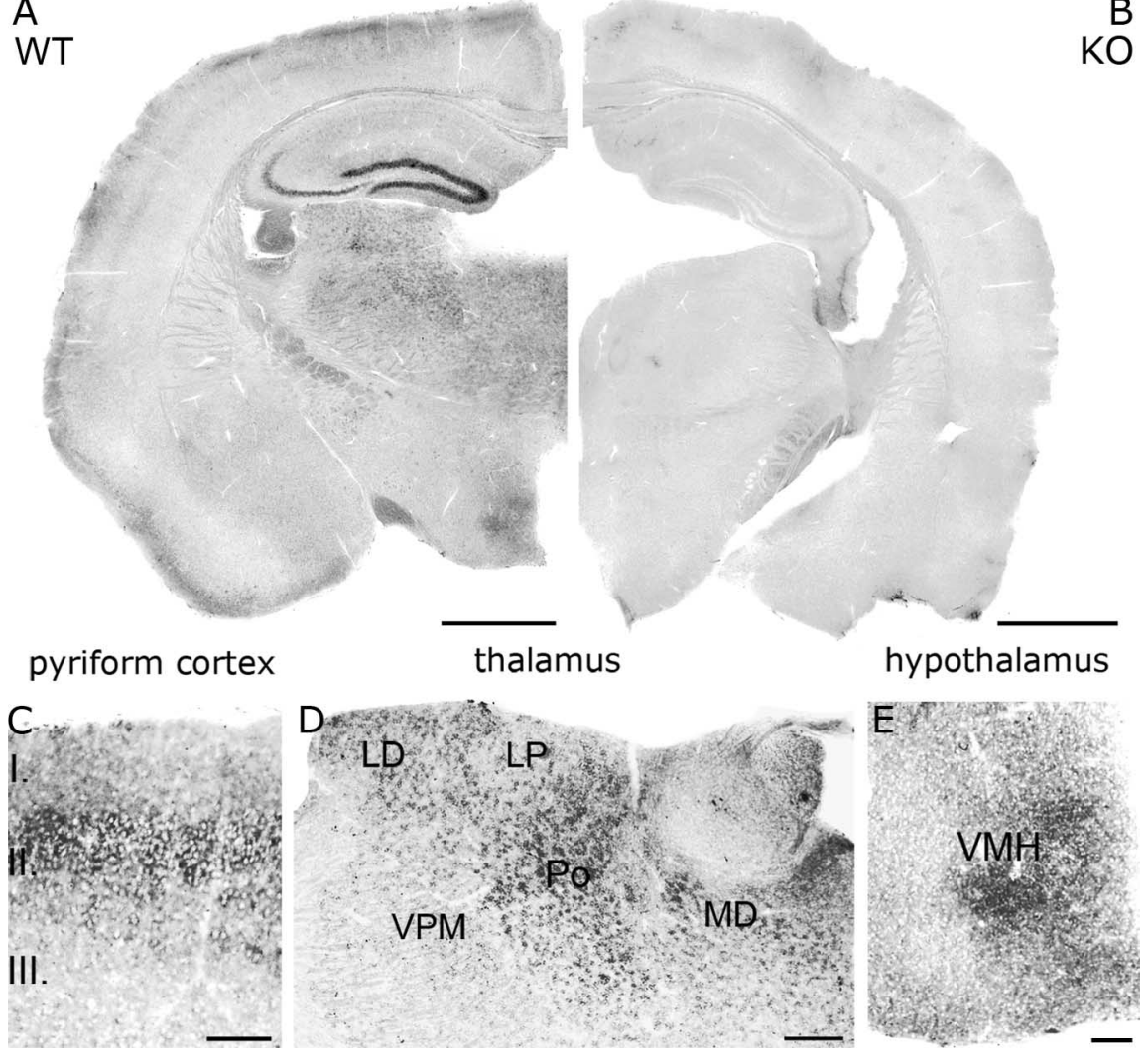

Figure 1. NAPE-PLD mRNA is expressed in selected brain regions at moderate-to-high levels. $\boldsymbol{A}$, In situ hybridization using a digoxigenin-labeled riboprobe against the mouse NAPE-PLD mRNA sequence highlights that granule cells in the dentate gyrus may have the highest expression of NAPE-PLD in the forebrain. Note that a relatively high expression level is also found in the CA3 pyramidal neurons, whereas modest labeling is visible in selected areas of the neocortex, the piriform cortex, the thalamus, and the hypothalamus. $\boldsymbol{B}$, In contrast, no labeling was found on sections derived from knock-out animals. Sections from wild-type and knock-out animals were mixed in the incubation wells and were processed together throughout the reactions. $\boldsymbol{C}-\boldsymbol{E}$, Highmagnification light micrographs show pyramidal neurons in layer II of the piriform cortex ( $\boldsymbol{C}$, neurons in higher-order thalamic nuclei $(\boldsymbol{D})$, and neurons in the ventromedial hypothalamic nuclei $(\mathrm{VMH} ; \boldsymbol{E})$. LD, Laterodorsal thalamic nucleus; LP, lateral posterior thalamic nucleus; Po, posterior thalamic nuclei; MD, mediodorsal thalamic nucleus; VPM, ventral posteromedial thalamic nucleus. Scale bars: $\boldsymbol{A}, \boldsymbol{B}, 1 \mathrm{~mm} ; \boldsymbol{C}, \boldsymbol{E}, 100 \mu \mathrm{m} ; \boldsymbol{D}, 200 \mu \mathrm{m}$.

behavioral activity profile, including the regulation of synaptic neurotransmission, has been documented for anandamide (Lovinger, 2007), as well as for other NAEs, which exhibit, for example, antiepileptic, antinociceptive ( $N$-palmitoylethanolamine), or anorexic ( $N$-oleoylethanolamine and $N$-stearoylethanolamine) effects (Okamoto et al., 2007). Despite their compelling functional importance in vivo, the underlying molecular architecture of anandamide and NAE synthesis in the brain has so far been elusive. To identify a potential source of anandamide and other NAEs at the cellular and subcellular level, we examined the distribution of $N$-acylphosphatidylethanolamine-hydrolyzing phospholipase D (NAPE-PLD), a recently identified synthetic enzyme of these bioactive lipids (Okamoto et al., 2007). NAPEPLD forms anandamide together with related NAE congeners by hydrolyzing their corresponding membrane precursors, the NAPEs, in a calcium-dependent manner (Di Marzo et al., 1994; Okamoto et al., 2007). Accordingly, we found that NAPE-PLD is associated with intracellular calcium stores in several types of excitatory axon terminals, indicating that, in contrast to 2-AG, endocannabinoids like anandamide and/or related NAEs may have a presynaptic origin and their production may reflect the status of axon terminal $\left[\mathrm{Ca}^{2+}\right]$ (in part following release from intracellular stores).

\section{Materials and Methods}

Perfusion and preparation of tissue sections. Experiments were performed according to the guidelines of the institutional ethical code and the Hungarian Act of Animal Care and Experimentation (1998; XXVIII. Section 243/1998). Adult male C57BL/6J mice [four wild-type and four NAPE-PLD knock-out (Leung et al., 2006), $77 \pm 30 \mathrm{~d}$ old] and a male $\mathrm{C} 57 \mathrm{BL} / 6 \mathrm{H}$ mouse (wild type, $60 \mathrm{~d}$ old) were deeply anesthetized with a mixture of ketamine-xylazine $(25 \mathrm{mg} / \mathrm{ml}$ ketamine, $5 \mathrm{mg} / \mathrm{ml}$ xylazine, $0.1 \% \mathrm{w} / \mathrm{w}$ pipolphen in $\mathrm{H}_{2} \mathrm{O} ; 1 \mathrm{ml} / 100$ g, i.p.). Animals were then perfused transcardially with $0.9 \%$ saline for $2 \mathrm{~min}$, followed by $100 \mathrm{ml}$ of fixative containing $4 \%$ paraformaldehyde in $0.1 \mathrm{~m}$ phosphate buffer (PB), pH 7.4, for $20 \mathrm{~min}$. The fixative of the $\mathrm{C} 57 \mathrm{BL} / 6 \mathrm{H}$ mouse also contained $0.5 \%$ glutaraldehyde. After perfusion, the brain was removed from the skull, postfixed for $2 \mathrm{~h}$ in the fixative, and washed in $\mathrm{PB}$, and then $50-\mu \mathrm{m}$ thick horizontal sections of the brain were cut with a Leica VTS-1000 vibratome (Vibratome, St. Louis, MO). For sections subjected to in situ hybridization, diethylpyrocarbonate-treated $\mathrm{PB}$ was used and sectioning was performed under RNase-free conditions.

In situ hybridization. The section of the mouse NAPE-PLD coding sequence (GenBank accession number, gi:142387907) were amplified by RT-PCR from cDNA derived from total C57BL/6J mouse frontal cortex mRNA. The length and the sequence of primers are listed below for the probe; numbering of the nucleotide positions starts from the beginning of the open reading frame. Probe: $1122 \mathrm{bp}$ from 31 to 1153 (forward primer, 5'-GCG CCA AGC TAT CAG TAT CC; reverse primer, 5'-TCT CGA CTC TCC ATG CTT CA). The primers were designed using the Primer3 software (Rozen and Skaletsky, 2000). Otherwise, the synthesis of riboprobes and the process of nonradioactive free-floating in situ hybridization were performed as published previously (Katona et al., 2006). The specificity of the riboprobe and the protocol was confirmed by the lack of reaction in forebrain sections derived from NAPE-PLD knock-out mice (Fig. $1 B$ ).

Peroxidase-based immunocytochemistry and preembedding immunogold labeling. For peroxidase-based immunocytochemistry, after extensive washing in $\mathrm{PB}$, sections from three wild-type mice and their corresponding NAPE-PLD knock-out littermates $(81 \pm 26 \mathrm{~d}$ old $)$, were first incubated for $10 \mathrm{~min}$ in $1 \% \mathrm{H}_{2} \mathrm{O}_{2}$ in $\mathrm{PB}$ to block endogenous peroxidase activity, then washed in $\mathrm{PB}$. For preembedding immunogold labeling, sections from three wild-type mice and their corresponding NAPE-PLD knock-out littermates ( $81 \pm 26 \mathrm{~d}$ old $)$ and a C57BL/6H wild-type mouse ( $60 \mathrm{~d}$ old) were used. Sections for both peroxidase-based immunocytochemistry and preembedding immunogold labeling were incubated in $30 \%$ sucrose overnight, followed by freeze thawing over liquid nitrogen four times and extensive washing in PB. Subsequently, all washing steps and dilutions of the antibodies were done in $0.05 \mathrm{M}$ Tris-buffered saline (TBS, pH 7.4). After washing in TBS, sections were blocked in 5\% normal goat serum for $45 \mathrm{~min}$, then incubated with either polyclonal affinitypurified guinea pig anti-NAPE-PLD (raised against the N-terminal 41 aa, $1: 1000$ to $1: 5000,0.72-0.14 \mu \mathrm{g} / \mathrm{ml}$ ) or rabbit anti-NAPE-PLD (raised against residues $38-53,1: 1000$ to $1: 5000, \sim 1-0.2 \mu \mathrm{g} / \mathrm{ml}$ ) (Fu et al., 2007) antibodies for $48 \mathrm{~h}$ at $4^{\circ} \mathrm{C}$. The specificity of both antibodies was confirmed by the lack of immunostaining in hippocampal sections derived from NAPE-PLD knock-out mice (Fig. $2 D$, supplemental Fig. $1 B$, avail- 


\section{NAPE-PLD mRNA}
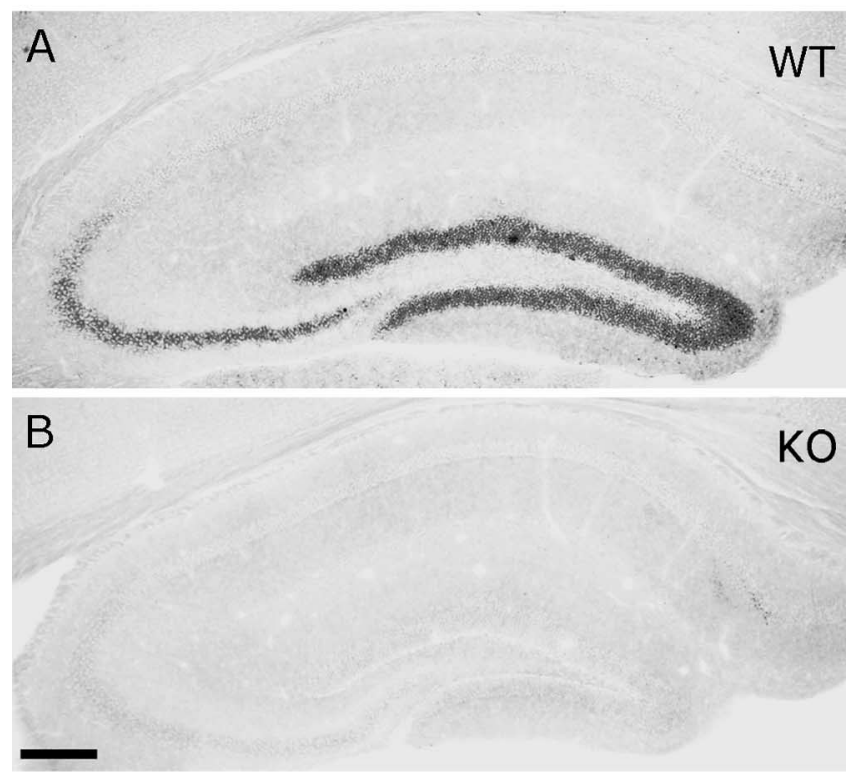

NAPE-PLD protein
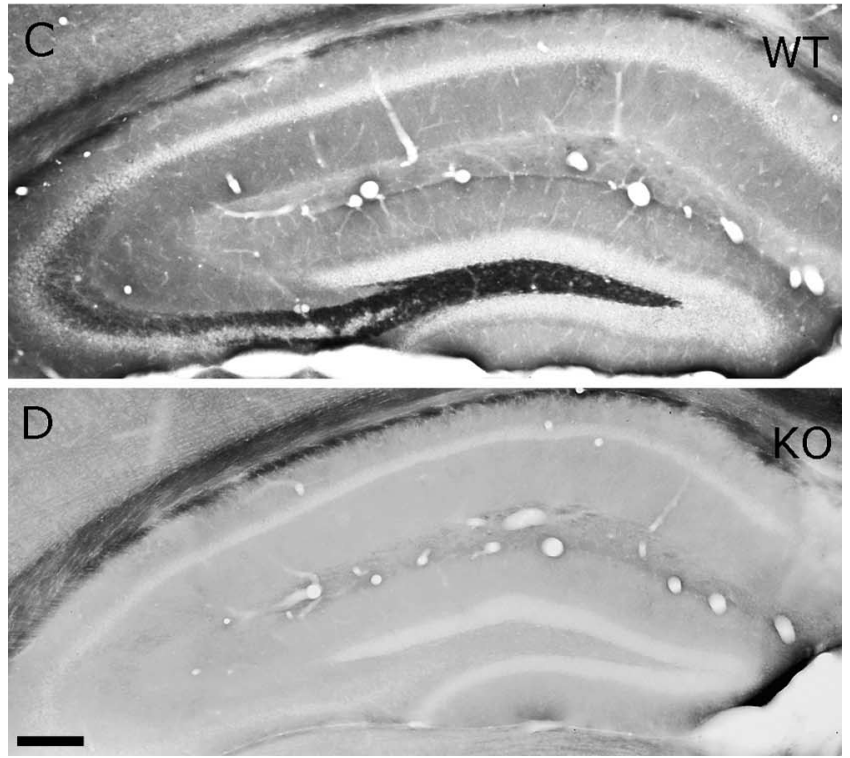

Figure 2. A, In situ hybridization reveals high levels of NAPE-PLD mRNA in granule cells and CA3 pyramidal neurons in wild-type animals. C, Immunocytochemistry for NAPE-PLD results in the strongest labeling in the hilus and in stratum lucidum, where mossy fibers arborize. $\boldsymbol{B}, \boldsymbol{D}$, The specificity of both reactions is confirmed in knock-out animals. Scale bars, $200 \mu \mathrm{m}$.

able at www.jneurosci.org as supplemental material). In the immunoperoxidase staining procedure, after primary antibody incubations and TBS washings, sections were treated with biotinylated goat anti-guinea pig IgG or with biotinylated goat anti-rabbit IgG (both 1:300; Vector Laboratories, Burlingame, CA) for $2 \mathrm{~h}$ and then, after washing with TBS, incubated with avidin-biotinylated horseradish peroxidase complex (1: 500; Elite-ABC; Vector Laboratories) for $1.5 \mathrm{~h}$. Peroxidase labeling was developed, after washings with TBS and $\mathrm{PB}$, using the $3,3^{\prime}$ diaminobenzidine (DAB)-nickel method. Sections were incubated in $\mathrm{PB}$ with $\mathrm{DAB}$ as chromogen $(0.298 \mathrm{mg} / \mathrm{ml})$, ammonium chloride $(0.396$ $\mathrm{mg} / \mathrm{ml})$, nickel-ammonium sulfate $(2.38 \mathrm{~mm})$, and $0.0005 \% \mathrm{H}_{2} \mathrm{O}_{2}$. The staining reaction was stopped by three washes with $\mathrm{PB}$. The sections were transferred onto glass slides from chrome gelatin, air dried, immersed in xylene, and mounted with Depex (SERVA Electrophoresis, Heidelberg, Germany).
In the immunogold staining procedure, sections were incubated in $0.8 \mathrm{~nm}$ gold-conjugated goat anti-guinea pig or goat anti-rabbit secondary antibody (both 1:50; AURION, Wageningen, The Netherlands), overnight at $4^{\circ} \mathrm{C}$. Then sections were silver intensified using the silver enhancement system R-GENT SE-EM according to the kit protocol (AURION). After silver intensification, sections were treated with $0.5 \% \mathrm{OsO}_{4}$ in $\mathrm{PB}$ for $20 \mathrm{~min}$ at $4^{\circ} \mathrm{C}$, dehydrated in an ascending series of ethanol and propylene oxide, and embedded in Durcupan (ACM, Fluka, Buchs, Switzerland). During dehydration, sections were treated with $1 \%$ uranyl acetate in $70 \%$ ethanol for $15 \mathrm{~min}$ at $4^{\circ} \mathrm{C}$. From sections embedded in Durcupan, areas of interest were reembedded and resectioned for electron microscopy. Ultrathin $(60 \mathrm{~nm})$ sections were collected on Formvarcoated single-slot grids and stained with lead citrate. Electron micrographs were taken at $20,000 \times, 30,000 \times$, and $70,000 \times$ magnifications with a Hitachi 7100 electron microscope (Tokyo, Japan).

Quantitative analysis of NAPE-PLD gold immunolabeling. For all quantitative analyses of immunogold labeling, we used EM sections labeled with guinea pig anti-NAPE-PLD antibody. NAPE-PLD immunoreactivity of presynaptic and postsynaptic elements in two hippocampal areas (hilus and CA1) was examined as a blind experiment on the corresponding sections of three wild-type and three NAPE-PLD knock-out littermate mice (100 profiles per area per animal). Fifteen mossy fiber boutons per animal were followed through five consecutive 60 -nm-thick serial sections in three wild-type mice, and the number of immunogold particles per each terminal within $300 \mathrm{~nm}$ in the $z$-axis direction was determined and normalized to a $1 \mu \mathrm{m}$ length for sake of easiness. To determine the subcellular distribution of NAPE-PLD, localization of immunogold particles was examined in excitatory boutons from the hilar region in four wild-type mice. Immunoparticles for NAPE-PLD were counted only in presynaptic and postsynaptic profiles that possessed clearly visible, anatomically defined asymmetrical synapses. Gold labeling was considered to be associated with a subcellular membrane structure if the gold particle was in contact with the membrane. Because a normal distribution could not be established (because of the small sample size), we used nonparametric statistics on our data. For the comparison of immunogold labeling in NAPE-PLD wild-type and knock-out mice, $\chi^{2}$ test was used. As animals within the same group (wild-type or $\mathrm{KO})$ were not significantly different $\left(\chi^{2}\right.$ test, $\left.p>0.05\right)$, data belonging to the same type of animals were pooled. In the case of the analysis of the subcellular distribution of gold particles within hilar excitatory boutons, two groups were compared using two-tailed nonparametric MannWhitney $U$ test. The differences were considered significant when the $p$ level was $<0.05$. For the statistical analyses, we used StatSoft (Tulsa, OK) Statistica software (version 7). Generation of bar graph was performed by Origin 7 software (OriginLab, Northampton, MA).

\section{Results}

Nonradioactive free-floating in situ hybridization on mouse forebrain sections revealed that granule cells of the dentate gyrus express high levels of NAPE-PLD mRNA (Figs. $1 A, 2 A$ ). In contrast, no labeling was observed on sections derived from knockout animals (Figs. 1B, 2B). A somewhat weaker but still high expression level was found in CA3 pyramidal neurons, whereas NAPE-PLD mRNA was not observed in CA1 pyramidal neurons, in hippocampal interneurons, and in glial cells (Figs. 1A, 2A), reflecting expression levels below the threshold of detection. Modest levels of NAPE-PLD mRNA were also observed in several other regions, e.g., in layers II-III of the neocortex, in layer II of the piriform cortex, in higher-order thalamic nuclei, and in the ventromedial hypothalamic nuclei (Fig. $1 A$ ). Because the highest amount of NAPE-PLD mRNA was found in the hippocampus, we focused our further analysis to this region. To determine the localization of NAPE-PLD protein, we used two independent antibodies, which stained identically in wild-type mice (Fig. 2C, supplemental Fig. $1 A$, available at www.jneurosci.org as supplemental material), but did not stain in knock-out mice (Fig. $2 D$, supplemental Fig. $1 B$, available at www.jneurosci.org as supple- 


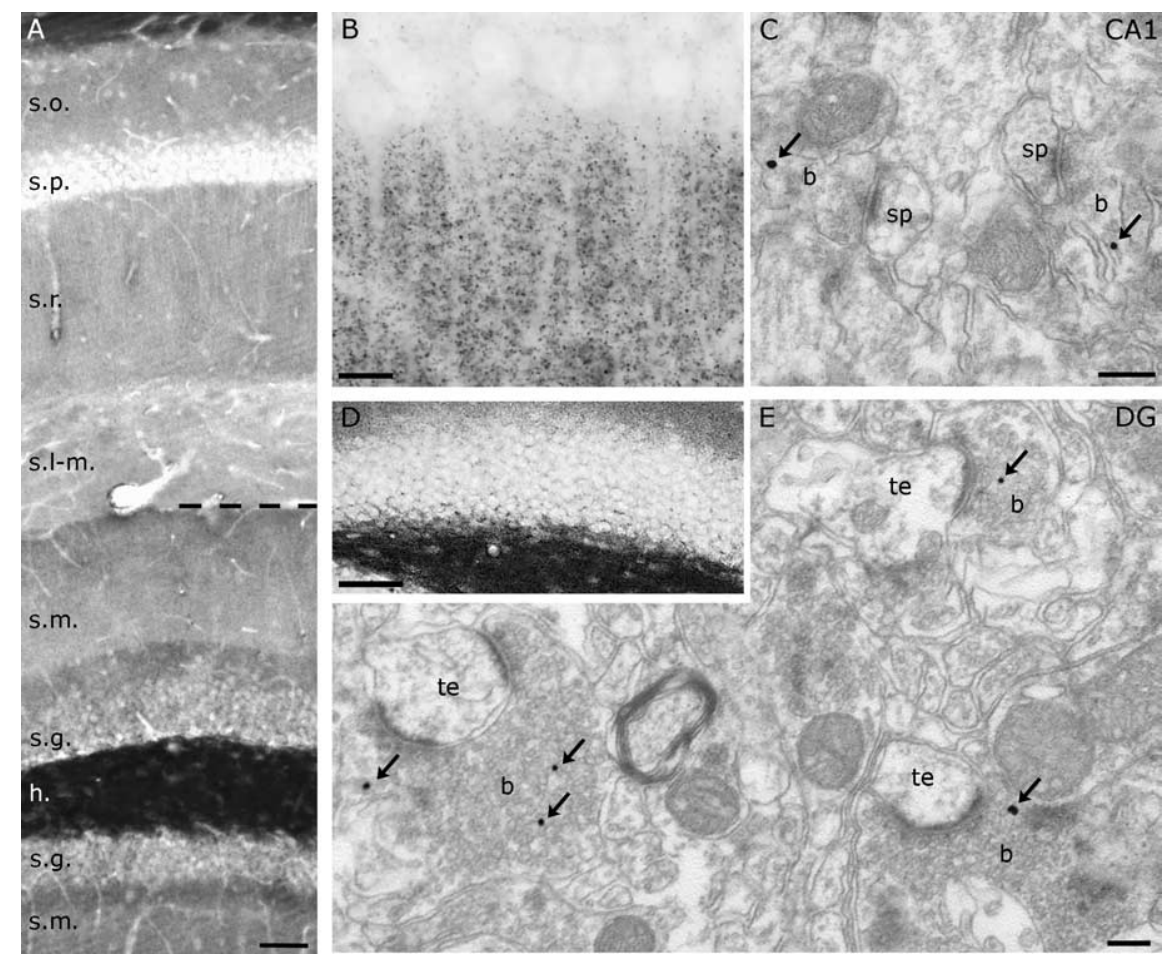

Figure 3. A, Immunocytochemistry for NAPE-PLD visualizes the laminar structure of the hippocampus, which is determined by the topography of excitatory pathways. The strongest labeling is found in the hilus (h) of the dentate gyrus. Dense NAPE-PLD immunoreactivity is present also in stratum moleculare (s.m.) (more profoundly in its inner third) and in the strata oriens (s.o.) and radiatum (s.r.) of the CA1 subfield, whereas in stratum lacunosum-moleculare (s-I.m.), immunostaining for NAPE-PLD did not reach detection threshold. $\boldsymbol{B}, \boldsymbol{D}$, At higher magnification, NAPE-PLD immunostaining reveals a dense punctate pattern in the CA1 stratum radiatum $(\boldsymbol{B})$ and a very dense punctate labeling in the hilus of the dentate gyrus (D). $\boldsymbol{C}, \boldsymbol{E}$, Immunogold particles (arrows) representing the precise subcellular localization of NAPE-PLD are found presynaptically in excitatory axon terminals both in the stratum radiatum (in Schaffer collaterals; $b$ in $\boldsymbol{C}$ ) and in the hilus (mossy terminals; b in $\boldsymbol{E}$ ), whereas postsynaptic structures [dendritic spines (sp) in $\boldsymbol{C}$ and thorny excrescences (te) in $\boldsymbol{E}$ ] are immunonegative. DG, Dentate gyrus; s.p., stratum pyramidale; s.g., stratum granulosum. Scale bars: $\boldsymbol{A}, \boldsymbol{D}, 50 \mu \mathrm{m} ; \boldsymbol{B}, 10 \mu \mathrm{m} ; \boldsymbol{C}, \boldsymbol{E}, 200 \mathrm{~nm}$.

mental material). In accordance with the high mRNA levels in dentate gyrus (Fig. 2A), immunoperoxidase staining for NAPEPLD visualized a dense meshwork of labeling in the hilus and stratum lucidum (Fig. $3 A, D$ ), which are the termination zones of mossy fibers, the axons of granule cells. A weaker but still characteristic NAPE-PLD staining followed the laminar pattern of other glutamatergic fibers (Fig. 3A). Importantly, the stratum (str.) radiatum of CA1 subfield, which is the termination zone of Schaffer collaterals, the axons of CA3 pyramidal cells, was also visualized (Fig. $3 A, B$ ), in accordance with the moderate NAPEPLD mRNA expression in these cells (Fig. 2A). At high magnification, immunostaining consisted of tiny granules homogenously distributed in the neuropil (Fig. $3 B, D$ ).

This immunostaining pattern and the in situ hybridization results suggest that NAPE-PLD is a presynaptic enzyme confined to glutamatergic axon terminals. To test this hypothesis, we performed high-resolution immunogold staining for NAPE-PLD at the electron microscopic level (EM). Quantitative analysis confirmed the presence of this enzyme in large mossy terminals in the hilus of wild-type but not knock-out animals (Fig. $3 E)(n=300-$ 300 axon terminals; $p<0.001$ ). Specific immunoreactivity was absent in their postsynaptic targets, the thorny excrescences (Fig. $3 E)(n=300-300$ postsynaptic profiles; $p=0.243)$. To estimate the density of NAPE-PLD labeling in boutons, mossy terminals were followed through serial sections for a distance of $300 \mathrm{~nm}$. Within this distance, $86.67 \%$ of the mossy terminals contained immunogold particles representing at least one NAPE-PLD mol- ecule (median value; range, 73.33-93.33\%; $n=45$ terminals, 3 animals). Considering that the diameter of mossy terminals ranges between 4 and $10 \mu \mathrm{m}$ (Acsady et al., 1998) and the density of gold particles within a bouton normalized to the diameter of the analyzed area was 6.22 particles/ $\mu \mathrm{m}$ (median value; range, 5.11-13.56), we can conclude that most if not all large mossy terminals contain NAPE-PLD (Fig. 3E). It is important to emphasize that beside the large mossy terminals, which target predominantly postsynaptic excitatory cells, small filopodia that contact exclusively GABAergic interneurons may also contain NAPE-PLD (Acsady et al., 1998). Unfortunately, axon terminals formed by these filopodia cannot be unequivocally distinguished from smaller mossy terminals at the electron microscopic level in absence of selective neurochemical markers for these terminal types. Nevertheless, to extend our observation to other glutamatergic synapses, we performed a similar analysis in stratum radiatum of the CA1 subfield, which revealed that Schaffer collaterals, but not their postsynaptic targets, also contain NAPEPLD (Fig. $3 C)(n=300-300$ profiles; $p=$ 0.00148 or $p=0.257$ for Schaffer collaterals or postsynaptic spines, respectively).

Previous immunogold EM studies have shown that the biosynthetic enzyme of 2-AG is selectively localized to the postsynaptic plasma membrane (Katona et al., 2006; Yoshida et al., 2006). In contrast, the present quantitative analysis of immunogold particle distribution at high resolution found that NAPE-PLD was not associated with the plasma membrane, but rather accumulated on the external surface of intracellular membrane compartments (Fig. $\left.4 A_{1}-B\right)$. Furthermore, the majority of gold particles were attached to cisternae of smooth endoplasmic reticulum (SER) (median, 58.08\%; range, $52.63-62 \% ; n=352$ gold particles; $n=4$ animals) (Fig. 4C).

\section{Discussion}

The present findings demonstrate that NAPE-PLD is a presynaptic enzyme in hippocampal glutamatergic axon terminals. This striking positioning of NAPE-PLD suggests that anandamide and other related NAEs are also synaptic signaling molecules, but, in contrast to the other major endocannabinoid 2-AG, they are not involved in retrograde synaptic communication, and are unlikely to act locally via $\mathrm{CB}_{1}$ cannabinoid receptors, which are absent from mossy terminals and undetectable on postsynaptic spines. Thus, an intriguing possibility is that these NAEs are acting on not yet characterized NAE receptors that may mediate novel forms of synaptic plasticity.

To elucidate the physiological significance of NAPE-PLD and its products at the synaptic level, one may consider at least two alternative routes for NAEs from their presynaptic origin. One possibility is that these bioactive lipids are released from glutamatergic axon terminals in an activity-dependent manner and act on postsynaptic targets. This anterograde route is indirectly sup- 
ported by the observation that fatty acid amide hydrolase (FAAH), the predominant enzyme for the inactivation of NAEs (Cravatt et al., 1996) is accumulated in the somatodendritic domain of postsynaptic principal cells (Gulyas et al., 2004); hence, NAEs must travel across the extracellular space to become inactivated. However, FAAH distribution is not entirely overlapping with NAPE-PLD; i.e., NAPE-PLD immunostaining has the highest density in str. lucidum (present study), whereas FAAH immunoreactivity is much weaker in this layer compared with other layers (Gulyas et al., 2004). Furthermore, immunoreactivity for NAPE-PLD did not reach detection threshold in the str. lacunosummoleculare, where a fine-grained immunostaining has been reported for FAAH (Gulyas et al., 2004). Another line of biochemical evidence suggests that FAAH is also responsible for the degradation of other structural classes of fatty acid amide signaling molecules (Saghatelian et al., 2006), whereas NAEs are degraded by additional brain enzymes beside FAAH (Tsuboi et al., 2007). Moreover, NAPEPLD-independent routes to generate anandamide and related NAEs have also been uncovered in brain tissue (Simon and Cravatt, 2006) and macrophage cells (Liu et al., 2006). Interestingly, one of these alternative biosynthetic enzymes, the $\alpha / \beta$ hydrolase 4, but not NAPE-PLD, shows evidence of coevolution with FAAH during phylogenesis (McPartland et al., 2007). This mismatch between the localization and evolutionary history of NAPE-PLD and FAAH together with the diversity of biosynthetic and catabolic pathways suggests that NAPE-PLD and its products may also be involved in alternative signaling routes.

In contrast to diacylglycerol lipase- $\alpha$, which is inserted into the plasma membrane and hydrolyzes plasma membrane phospholipid precursors to the intercellular signal molecule 2-AG, NAPE-PLD is accumulated on the surface of intracellular membranous structures. These organelles (SERs) may also serve as lipid reservoirs and indeed, NAPE-PLD catalytic activity as well as the precursor NAPE molecules are both found in purified microsome fractions, but not in brain plasma membrane fractions (Cadas et al., 1997; Okamoto et al., 2007). Moreover, NAPE-PLD catalytic activity is calcium dependent, and presynaptic SERs have a well known role as calcium stores in axon terminals; for example, calcium release from these internal stores contributes to the short-term facilitation of transmitter release (Emptage et al., 2001). Importantly, van der Stelt et al. (2005) have shown in dorsal root ganglia neurons that intracellular calcium mobilization from these stores triggers anandamide biosynthesis and results in amplification of calcium influx via TRPV1 channels. Together with the present findings, these observations raise also the possibility of an intracellular role for anandamide and/or related NAEs. Irrespectively of the intercellular or intracellular routes (which may even coexist, if NAPE-PLD synthe- sizes more than one species of NAE within one axon terminal), further progress requires that the authentic targets of these bioactive lipids be identified from the plethora of receptor candidates found in heterologous expression systems. The precise cellular and subcellular source of anandamide and its related bioactive congeners revealed in this study may help to focus these efforts to native systems such as the mossy fiber synapse and adds a new dimension to endocannabinoid functions in synaptic signaling.

\section{References}

Acsady L, Kamondi A, Sik A, Freund T, Buzsaki G (1998) GABAergic cells are the major postsynaptic targets of mossy fibers in the rat hippocampus. J Neurosci 18:3386-3403.

Cadas H, di Tomaso E, Piomelli D (1997) Occurrence and biosynthesis of endogenous cannabinoid precursor, $N$-arachidonoyl phosphatidylethanolamine, in rat brain. J Neurosci 17:1226-1242.

Chevaleyre V, Takahashi KA, Castillo PE (2006) Endocannabinoidmediated synaptic plasticity in the CNS. Annu Rev Neurosci 29:37-76.

Cravatt BF, Giang DK, Mayfield SP, Boger DL, Lerner RA, Gilula NB (1996) Molecular characterization of an enzyme that degrades neuromodulatory fatty-acid amides. Nature 384:83-87.

Devane WA, Hanus L, Breuer A, Pertwee RG, Stevenson LA, Griffin G, Gibson D, Mandelbaum A, Etinger A, Mechoulam R (1992) Isolation and structure of a brain constituent that binds to the cannabinoid receptor. Science 258:1946-1949. 
Di Marzo V, Fontana A, Cadas H, Schinelli S, Cimino G, Schwartz JC, Piomelli D (1994) Formation and inactivation of endogenous cannabinoid anandamide in central neurons. Nature 372:686-691.

Di Marzo V, Bisogno T, De Petrocellis L (2007) Endocannabinoids and related compounds: walking back and forth between plant natural products and animal physiology. Chem Biol 14:741-756.

Emptage NJ, Reid CA, Fine A (2001) Calcium stores in hippocampal synaptic boutons mediate short-term plasticity, store-operated Ca2+ entry, and spontaneous transmitter release. Neuron 29:197-208.

Fu J, Astarita G, Gaetani S, Kim J, Cravatt BF, Mackie K, Piomelli D (2007) Food intake regulates oleoylethanolamide formation and degradation in the proximal small intestine. J Biol Chem 282:1518-1528.

Gulyas AI, Cravatt BF, Bracey MH, Dinh TP, Piomelli D, Boscia F, Freund TF (2004) Segregation of two endocannabinoid-hydrolyzing enzymes into pre- and postsynaptic compartments in the rat hippocampus, cerebellum and amygdala. Eur J Neurosci 20:441-458.

Hashimotodani Y, Ohno-Shosaku T, Kano M (2007) Presynaptic monoacylglycerol lipase activity determines basal endocannabinoid tone and terminates retrograde endocannabinoid signaling in the hippocampus. J Neurosci 27:1211-1219.

Katona I, Urban GM, Wallace M, Ledent C, Jung KM, Piomelli D, Mackie K, Freund TF (2006) Molecular composition of the endocannabinoid system at glutamatergic synapses. J Neurosci 26:5628-5637.

Kim J, Alger BE (2004) Inhibition of cyclooxygenase-2 potentiates retrograde endocannabinoid effects in hippocampus. Nat Neurosci 7:697-698.

Leung D, Saghatelian A, Simon GM, Cravatt BF (2006) Inactivation of $\mathrm{N}$-acyl phosphatidylethanolamine phospholipase D reveals multiple mechanisms for the biosynthesis of endocannabinoids. Biochemistry 45:4720-4726.

Liu J, Wang L, Harvey-White J, Osei-Hyiaman D, Razdan R, Gong Q, Chan AC, Zhou Z, Huang BX, Kim HY, Kunos G (2006) A biosynthetic pathway for anandamide. Proc Natl Acad Sci USA 103:13345-13350.
Lovinger DM (2007) Endocannabinoid liberation from neurons in transsynaptic signaling. J Mol Neurosci 33:87-93.

Makara JK, Mor M, Fegley D, Szabo SI, Kathuria S, Astarita G, Duranti A, Tontini A, Tarzia G, Rivara S, Freund TF, Piomelli D (2005) Selective inhibition of 2-AG hydrolysis enhances endocannabinoid signaling in hippocampus. Nat Neurosci 8:1139-1141.

McPartland JM, Norris RW, Kilpatrick CW (2007) Coevolution between cannabinoid receptors and endocannabinoid ligands. Gene 397:126-135.

Okamoto Y, Wang J, Morishita J, Ueda N (2007) Biosynthetic pathways of the endocannabinoid anandamide. Chem Biodivers 4:1842-1857.

Piomelli D, Astarita G, Rapaka R (2007) A neuroscientist's guide to lipidomics. Nat Rev Neurosci 8:743-754

Rozen S, Skaletsky H (2000) Primer3 on the WWW for general users and for biologist programmers. Methods Mol Biol 132:365-386.

Saghatelian A, McKinney MK, Bandell M, Patapoutian A, Cravatt BF (2006) A FAAH-regulated class of $\mathrm{N}$-acyl taurines that activates TRP ion channels. Biochemistry 45:9007-9015.

Simon GM, Cravatt BF (2006) Endocannabinoid biosynthesis proceeding through glycerophospho- $\mathrm{N}$-acyl ethanolamine and a role for alpha/betahydrolase 4 in this pathway. J Biol Chem 281:26465-26472.

Tsuboi K, Takezaki N, Ueda N (2007) The N-acylethanolaminehydrolyzing acid amidase (NAAA). Chem Biodivers 4:1914-1925.

van der Stelt M, Trevisani M, Vellani V, De Petrocellis L, Schiano Moriello A Campi B, McNaughton P, Geppetti P, Di Marzo V (2005) Anandamide acts as an intracellular messenger amplifying Ca2+ influx via TRPV1 channels. EMBO J 24:3026-3037.

Wilson RI, Nicoll RA (2002) Endocannabinoid signaling in the brain. Science 296:678-682.

Yoshida T, Fukaya M, Uchigashima M, Miura E, Kamiya H, Kano M, Watanabe M (2006) Localization of diacylglycerol lipase- $\alpha$ around postsynaptic spine suggests close proximity between production site of an endocannabinoid, 2-arachidonoyl-glycerol, and presynaptic cannabinoid CB1 receptor. J Neurosci 26:4740-4751. 